\title{
Synergie potentielle entre deux toxiques rénaux : le DTPA et l'uranium
}

\author{
D. MULLER ${ }^{1,2}$, P. HOUPERT ${ }^{1}$, M.H. HENGE NAPOLI ${ }^{1,4}$, H. MÉTIVIER $^{3}$, \\ F. PAQUET ${ }^{1}$
}

(Manuscrit reçu le 21 avril 2006, accepté le 25 juin 2006)

RÉSUMÉ Le traitement recommandé en cas de contamination accidentelle par un composé hybride d'oxydes de plutonium et d'uranium (MOX) est l'administration d'acide diethylène-triamine-pentaacétate (DTPA) afin d'accélérer l'excrétion du plutonium. L'uranium et le DTPA étant deux composés néphrotoxiques, l'administration de DTPA consécutif à une contamination par un composé contenant de l'uranium, est susceptible de potentialiser les effets néphrotoxiques de l'uranium. L'objectif de ce travail a été d'étudier in vitro sur une lignée de cellules tubulaires proximales de rein (LLC-PK $)_{1}$ la cytotoxicité induite par des concentrations croissantes d'uranium en présence ou en absence de 3 formes chimiques de DTPA. Les résultats ont montré que le DTPA, utilisé seul, n'induisait aucune toxicité à la concentration testée $(420 \mu M)$. Cependant, l'administration de DTPA augmentait le taux de mortalité cellulaire induit par l'uranium. Cette augmentation était maximale pour les concentrations en uranium proches de la concentration létale pour $50 \%$ des cellules et atteignait respectivement 37,31 et $28 \%$ pour le DTPA anhydre, le $\mathrm{Na}_{3} \mathrm{CaDTPA}$ et le $\mathrm{Na}_{3} \mathrm{ZnDTPA}$. Ces résultats suggèrent que le DTPA est un composé susceptible de potentialiser la néphrotoxicité induite par l'uranium.

ABSTRACT Potential synergy between two renal toxicants: DTPA and uranium.

At present, the most appropriate therapeutic approach to treat an accidental contamination with plutonium and uranium oxyde mixture (MOX) is administration of diethylene-triamine-pentaacetate acid (DTPA) in order to accelerate plutonium excretion. As uranium and DTPA are both nephrotoxic compounds, the administration of DTPA after a contamination containing uranium could enhance the nephrotoxic effects of uranium. The aim of the present work was to study in vitro on a kidney proximal tubule cell line (LLC-PK $)_{1}$ the cytotoxicity induced by increasing concentrations of uranium in presence of 3 different chemical forms of DTPA. The results showed that the DTPA used alone induced no cytotoxicity at the concentration used here $(420 \mu \mathrm{M})$. However, this concentration of DTPA increased the cytotoxicity induced by uranium. This increase was maximal for uranium concentrations close to the lethal concentration for $50 \%$ of the cells and reached 37,31 and $28 \%$ for anhydrous DTPA, $\mathrm{Na}_{3} \mathrm{CaDTPA}$ and $\mathrm{Na}_{3} \mathrm{ZnDTPA}$, respectively. These results suggest that administration of DTPA could enhance the nephrotoxicity induced by uranium.

Keywords: uranium / DTPA / kidney / toxicity / in vitro

1 IRSN, Direction de la radioprotection de l'homme, Service de radiobiologie et d'épidémiologie, Laboratoire de radiotoxicologie expérimentale, B.P. 166, 26702 Pierrelatte, France.

2 Adresse actuelle : King's College London, School of Health and Biomedical Sciences Division of Reproductive Health, Endocrinology and Development Beta Cell Function and Development Group Hodgkin Building, London SE1 1UL, UK.

3 IRSN, B.P. 17, 92262 Fontenay-aux-Roses Cedex, France.

4 Adresse actuelle : CEA/DEN/Valrhô-Dir-CVAR, B.P. 17171, 30207 Bagnols-sur-Cèze Cedex, France. 


\section{Introduction}

En cas de contamination interne, il est recommandé d'utiliser des chélateurs afin d'accélérer l'excrétion des radionucléides et ainsi de réduire les doses délivrées à l'organisme. À l'heure actuelle, les médecins ne disposent d'aucun produit performant pour traiter une contamination accidentelle par des composés hybrides d'oxydes d'uranium et de plutonium (MOX) couramment manipulés aux postes de travail de nombreuses usines du cycle du combustible. Le seul traitement proposé est le DTPA (acide diethylène-triamine-pentaacétate), utilisé pour traiter les contaminations au plutonium, alors que son efficacité pour augmenter l'excrétion $\mathrm{du}$ plutonium incorporé sous forme de MOX est encore mal connue (Bhattacharyya et al., 1995; Wood et al., 2000). De plus le DTPA est néphrotoxique lorsqu'il est administré en grande quantité (Doolan et al., 1967 ; Morgan et Smith, 1974). L'uranium étant également néphrotoxique dès qu'il atteint une concentration de $3 \mu \mathrm{g} / \mathrm{g}$ de rein (Diamond et al., 1989), la question est de savoir si le DTPA, administré pour réduire les risques dus au plutonium, ne risque pas de potentialiser la néphrotoxicité induite par l'uranium. L'objectif de cette étude est d'apporter un premier élément de réponse. Cette étude a été réalisée in vitro sur une lignée de cellules tubulaires proximales de rein, tissu cible de la toxicité de l'uranium (Diamond et al., 1989). La survie des cellules a été évaluée après 24 heures d'incubation en présence d'uranium et de DTPA.

\section{Matériels et méthodes}

\subsection{Réactifs et matériels}

Le sérum de veau fœtal (SVF), l'HEPES, les flacons de cultures, la L-glutamine, la streptomycine et la pénicilline ont été obtenus chez Sigma (l'Isle d'Abeau Chesnes, France) et le nitrate d'uranyl chez Merk Eurolab (Lyon, France). Le DTPA anhydre a été obtenu chez Sigma (l'Isle d'Abeau Chesnes, France), tandis que les formes zincique et calcique de DTPA ont été obtenues respectivement par le service médical du travail de COGEMA (Pierrelatte, France) et par le Service de protection radiologique des Armées (Clamart, France). Les cellules LLC-PK 1 ont été obtenues à l'European Collection of Cell Culture (Wilshire, Angleterre).

\subsection{Culture cellulaire}

La croissance cellulaire a été effectuée en milieu de culture nutritionnel supplémenté de $5 \%$ de sérum de veau fotal (SVF), $10 \mathrm{mM}$ d'HEPES, $1 \mathrm{mM}$ de L-glutamine, $50 \mathrm{mg} / \mathrm{l}$ de streptomycine et $10^{4} \mathrm{UI} / 1$ de pénicilline en atmosphère humide composée de $5 \%$ de $\mathrm{CO}_{2}$ et de $95 \%$ d'air à $37^{\circ} \mathrm{C}$. À confluence, 
les cellules ont été détachées et séparées mécaniquement avant d'être repiquées soit à une densité de $10^{6}$ cellules par flacon de culture soit à une densité de $10^{3}$ par puits sur plaque de micro-titration.

\subsection{Préparation des solutions d'uranium}

Les cristaux de nitrate d'uranium ont été dissous dans une solution de bicarbonate à $0,1 \mathrm{M}$ afin d'obtenir une solution mère en uranium de $10 \mathrm{mM}$ (Mirto et al., 1999). À partir de cette solution, une gamme de concentration a été réalisée en milieu bicarbonate $0,1 \mathrm{mM}$. Les solutions obtenues ont été ajustées à pH 7,4.

\section{4. Études de cytotoxicité}

Les cellules ont été amenées à confluence dans des puits de plaque de microtitration en milieu complémenté avec $10 \%$ de SVF. Elles ont alors été incubées avec une gamme de concentrations croissantes en uranium pendant 24 heures, en présence ou en absence de DTPA anhydre, de $\mathrm{Na}_{3} \mathrm{CaDTPA}$ ou de $\mathrm{Na}_{3} \mathrm{ZnDTPA}$ à la concentration de $420 \mu \mathrm{M}$. Cette concentration est une extrapolation de la posologie employée en cas de contamination humaine par le plutonium. En effet, l'injection de $30 \mu \mathrm{mol} / \mathrm{kg}$ de DTPA à un homme moyen possédant un volume sanguin de 5 litres conduit à une concentration sanguine immédiate de $420 \mu \mathrm{M}$ de DTPA. Le taux de lactate déhydrogénase (LDH) relarguée dans le milieu par les cellules endommagées a été mesuré à l'aide d'un kit de détection enzymatique (Boerhinger Manheim, Allemagne). La consommation de nicotine adénine dinucléotide en présence de pyruvate a été évaluée par spectrométrie d'absorbance à $490 \mathrm{~nm}$. Le taux de cytotoxicité a été calculé à partir des densités optiques à l'aide de la formule suivante :

Taux de cytotoxicité $=\frac{(\text { DO cellules testées }- \text { DO temoin négatif })}{(\text { DO témoin positif }- \text { DO temoin négatif })} \times 100$.

\section{Résultats}

\subsection{Toxicocinétique de l'uranium}

En présence d'une concentration de $1 \mathrm{mM}$ d'uranium, le taux de LDH relargué dans le milieu extracellulaire par les cellules endommagées augmente pendant les premières heures d'incubation pour atteindre $100 \%$ en 20 à 25 heures (Fig. 1). La mortalité cellulaire étant alors maximale, les études ultérieures ont été réalisées après 24 heures d'incubation. 


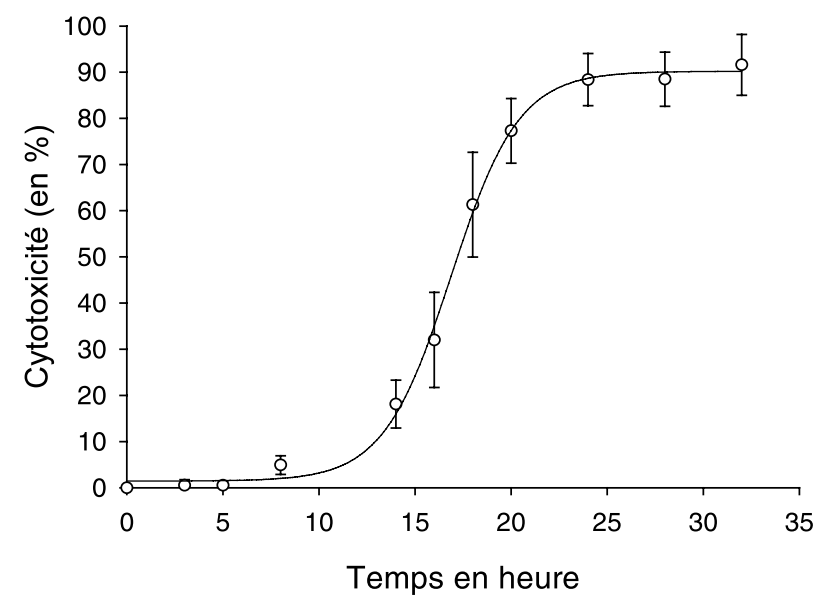

Figure 1 - Toxicocinétique de l'uranium (1 mM) sur les cellules LLC-PK . Uranium toxicokinetics on LLC-PK cells.

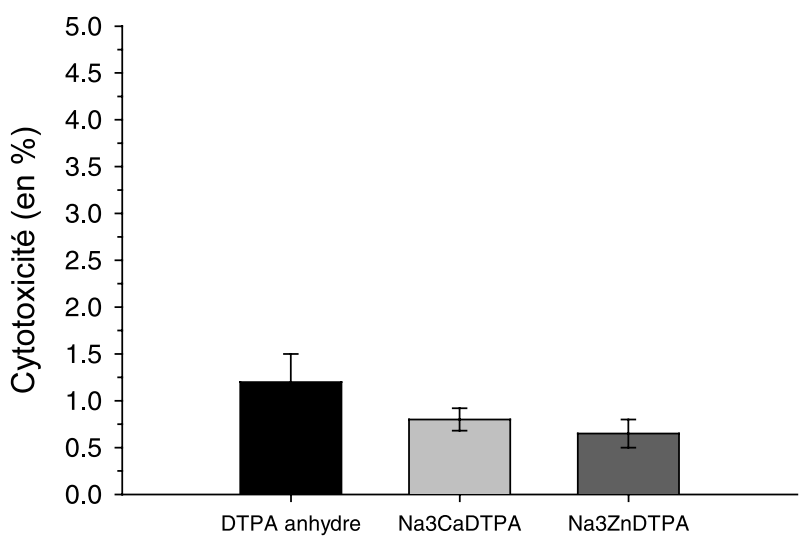

Figure 2 - Le DTPA sous forme anhydre, calcique ou zincique à la concentration de $420 \mu \mathrm{M} n$ 'induit pas de cytotoxicité significative sur les cellules $L L C-P K_{1}$ en 24 heures d'incubation.

Anhydrous, calcium or zinc salts of DTPA used at a concentration of $420 \mu M$ did not induce any significant toxicity on $L L C$-PK $K_{1}$ cells after $24 \mathrm{~h}$ of incubation.

\subsection{Potentialisation de la cytotoxicité de l'uranium par le DTPA}

L'incubation pendant 24 heures des cellules LLC-PK1 en présence de $420 \mu \mathrm{M}$ de DTPA, qu'il soit sous forme anhydre, calcique ou zincique, n'a pas induit d'effet cytotoxique significatif (Fig. 2). 


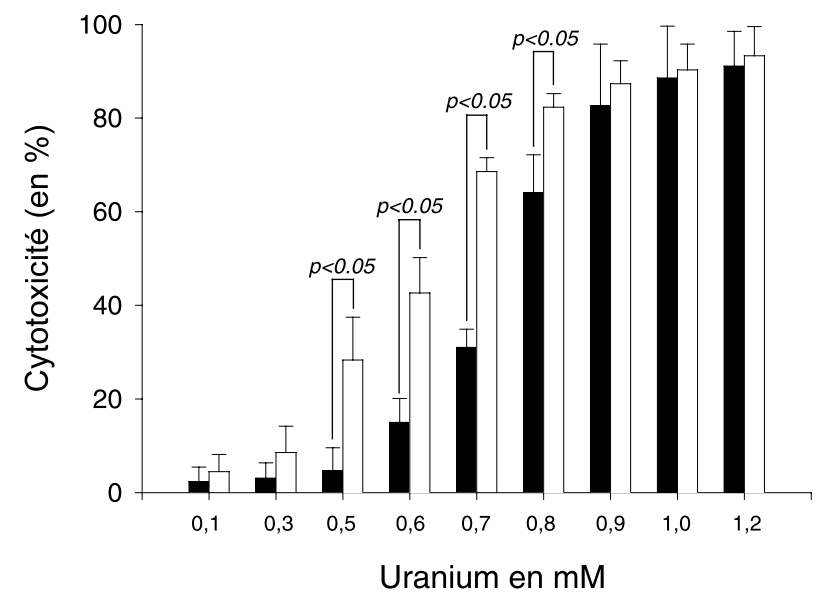

Figure 3 - Le DTPA anhydre à la concentration de $420 \mu M$ augmente la cytotoxicité induite par l'uranium sur les cellules $L L C-P K_{1}$ après 24 heures d'incubation ( $\square$ : uranium, $\square:$ uranium + DTPA). Anhydrous DTPA used at a concentration of $420 \mu M$ increased the uranium cytotoxicity on LLC-PK ${ }_{1}$ cells after $24 \mathrm{~h}$ of incubation.

En revanche, lorsqu'il est associé avec l'uranium, le DTPA a augmenté significativement le taux de mortalité cellulaire et ce pour les 3 formes chimiques de DTPA testées (Figs. 3 à 5). Cette augmentation était maximale pour les concentrations en uranium proches de la concentration létale pour $50 \%$ des cellules qui se situait aux alentours de $700 \mu \mathrm{M}$ d'uranium. L'augmentation de la toxicité de l'uranium était alors de 37,5, 30,9 et 27,8\% respectivement pour le DTPA anhydre, le $\mathrm{Na}_{3} \mathrm{CaDTPA}$ et le $\mathrm{Na}_{3} \mathrm{ZnDTPA}$.

\section{Discussion}

L'objectif de cette étude était de tester in vitro sur culture cellulaire une synergie potentielle de l'uranium et du DTPA sur la toxicité rénale. Les cultures cellulaires sont en effet largement utilisées dans l'étude des processus physiopathologiques des molécules thérapeutiques ou des toxiques (Takano et al., 1994 ; Endo et al., 1996 ; Decorti et al., 1999 ; Choi et al., 1999). Les deux modèles de cellules tubulaires proximales de rein les plus fréquemment utilisées dans ces études sont les lignées cellulaires immortalisées $\mathrm{LLC}_{\mathrm{PK}}$ et OK. Les précédentes études in vitro de toxicité de l'uranium ayant été effectuées sur la lignée cellulaire LLC-PK 1 (Mirto et al., 1999), nous avons choisi ce modèle pour notre étude. 


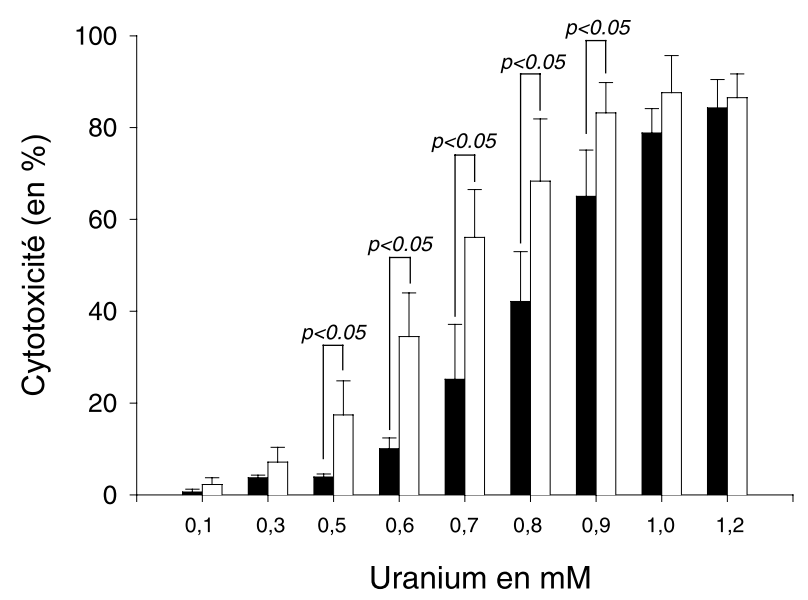

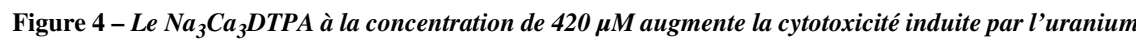
sur les cellules LLC-PK 1 après 24 heures d'incubation (ロ : uranium, $\square$ : uranium +DTPA). Calcium salts of DTPA used at a concentration of $420 \mu M$ increased the uranium cytotoxicity

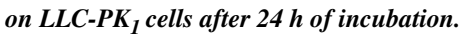

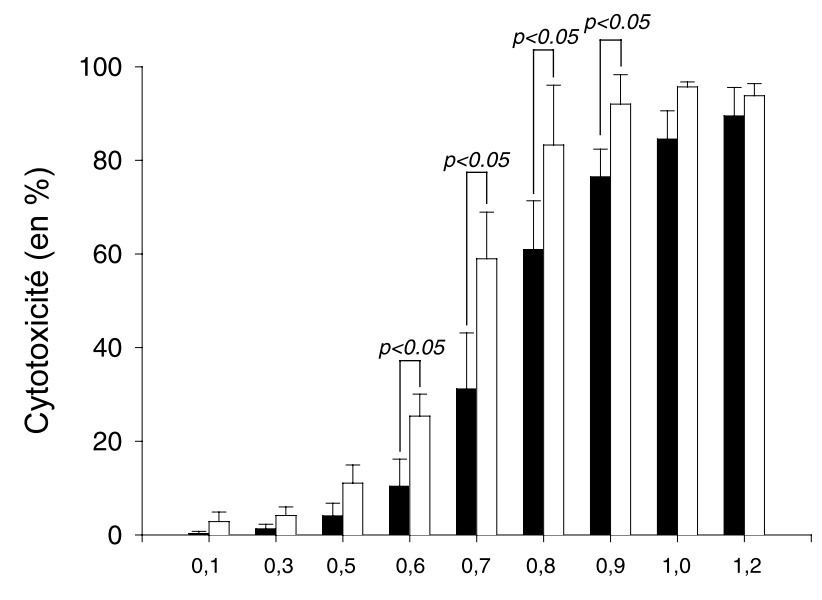

Uranium en $\mathrm{mM}$

Figure 5 - Le $\mathrm{Na}_{3} \mathrm{ZnDTPA}$ à la concentration de $420 \mu \mathrm{M}$ augmente la cytotoxicité induite par l'uranium sur les cellules $L L C$-PK 1 après 24 heures d'incubation ( $:$ :uranium, $\square:$ uranium + DTPA). Zinc salts of DTPA used at a concentration of $420 \mu M$ increased the uranium cytotoxicity on

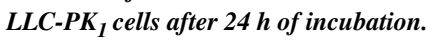


Le premier résultat de notre étude a montré que le DTPA à une concentration de $420 \mu \mathrm{M}$, équivalente à la concentration thérapeutique utilisée chez l'homme, quelle que soit la forme chimique utilisée (DTPA anhydre, $\mathrm{Na}_{3} \mathrm{CaDTPA}$ et le $\mathrm{Na}_{3} \mathrm{ZnDTPA}$ ), n'engendre pas d'effets cytotoxiques après une période d'incubation de 24 heures. Ces résultats, obtenus in vitro, sont en accord avec les résultats de la littérature obtenus in vivo. En effet, in vivo le DTPA n'est toxique que pour de fortes concentrations (Doolan et al., 1967) et à la dose thérapeutique de $30 \mu \mathrm{mol} / \mathrm{kg}$, il n'altère ni les fonctions ni la cytologie des reins ou du foie chez le singe (Fritsch et al., 1994).

Le second résultat de notre étude a montré que la cytotoxicité de l'uranium était augmentée par le DTPA. Pour des concentrations de $700 \mu \mathrm{M}$ d'uranium, voisines de la concentration létale pour $50 \%$ des cellules, l'adjonction de DTPA dans le milieu de culture a augmenté la mortalité cellulaire d'environ $30 \%$. Or le DTPA seul n'induisant pas d'effet toxique, cette augmentation de cytotoxicité peut s'expliquer soit par une potentialisation de la toxicité de l'uranium par le DTPA, soit par la chélation d'éléments essentiels à l'homéostasie cellulaire, tels le zinc, le manganèse ou le calcium (Dvorak, 1970; Havlicek, 1967). Cette dernière hypothèse semble peu probable. En effet, le milieu de culture utilisé était dépourvu de zinc et de manganèse et de plus, les formes zincique et calcique de DTPA induisaient des effets tout à fait similaires à ceux de la forme anhydre de DTPA. Il semble donc plus probable que le DTPA potentialise un ou plusieurs mécanismes cellulaires impliqués dans la toxicité de l'uranium.

En conclusion, cette étude montre que le DTPA augmente la mortalité cellulaire induite par l'uranium sur une lignée de cellules tubulaires proximales de rein, la lignée $L L C-\mathrm{PK}_{1}$. Ces résultats suggèrent une potentialisation de la néphrotoxicité de l'uranium par le DTPA. Cependant, avant de déconseiller l'utilisation du DTPA pour traiter une contamination au MOX, ces résultats devront être confirmés in vivo afin de prendre en compte les biocinétiques respectives de l'uranium et du DTPA. En effet, les transformations physicochimiques subies par l'uranium et le DTPA avant d'atteindre les cellules tubulaires rénales ainsi que le temps de contact entre les cellules tubulaires rénales et l'uranium ou le DTPA pourraient moduler les résultats obtenus in vitro.

\section{RÉFÉRENCES}

Bhattacharyya M.H., Breitenstein B.D., Métivier H., Stradling G.N., Volf V. (1995) Traitement de la contamination interne accidentelle des travailleurs. Rapport IPSN, Fontenay-aux-Roses.

Choi J.S., Kim K.R., Ahm D.W., Park Y.S. (1999) Cadmium inhibits albumin endocytosis in opossum kidney epithelial cells, Toxicol. Appl. Pharm. 161, 146-152.

Decorti G., Malusà N., Furlan G., Candussio L., Klugmann F.B. (1999) Endocytosis of gentamicin in a proximal tubular renal cell line, Life Sci. 65, 1115-1124. 
Diamond G.L., Morrow P.E., Panner B.J., Gelein R.M., Baggs R.B. (1989) Reversible uranyl fluride nephrotoxicity in the Long Evans rat. Fund. Appl. Toxicol. 13, 65-78.

Doolan P.D., Schwartz S.L., Hayes J.R., Mullen J.C., Cummings N.B. (1967) An evaluation of the nephrotoxicity of ethylenediaminetetraacetate and diethylenetriaminepentaacetate in the rat, Tox. Appl. Pharmacol. 10, 481-500.

Dvorak P. (1970) Metabolism and toxicity of therapeutic agents. 8. Excretion of sodium, potassium, magnesium and zinc, Strahlentherapie 139, 611-618.

Endo T., Kimura O., Sakata M. (1996) Comparative studies of cadmium and mercury accumulation by LLC-PK $_{1}$ cells: effects of $\mathrm{pH}$ on uptake and efflux, Toxicol. Lett. 87, 77-83.

Fritsch P., Herbreteau D., Moutairou K., Lantenois G., Richard-Le Naour H., Grillon G., Hoffschir D., Poncy J.L., Laurent A., Masse R. (1994) Comparative toxicity of 3,4,3-LIHOPO and DTPA in baboons: preliminary results, Radiat. Prot. Dosim. 53, 315-318.

Havlicek F. (1967) Metabolism and toxicity of therapeutic chelating agents. 3. Effect of EDTA and DTPA on the excretion of endogenous zinc, Strahlentherapie 134, 296-305.

Mirto H., Hengé-Napoli M.H., Gibert R., Ansoborlo E., Fournier M., Cambar J. (1999) Intracellular behaviour of uranium (VI) on renal epithelial cell in culture $\left(\mathrm{LLC}_{\mathrm{PK}}\right)$ : influence of uranium speciation, Toxicol. Lett. 104, 249-256.

Morgan R.M., Smith H. (1974) Histological changes in kidney, liver and duodenum of the mouse following the acute and subacute administration of diethylenetriaminepentaacetic acid, Toxicology 2, 153-163.

Takano M., Ohishi Y., Okuda M., Yasuhara M., Hori R. (1994) Transport of gentamincin and fluidphase endocytis markers in the LLC-PK 1 kidney epithelial cell line, J. Pharmacol. Exp. Ther. 268, 669-673.

Wood R., Sharp C., Gourmelon P., Le Guen B., Stradling G.N., Taylor D.M., Hengé-Napoli M.H. (2000) Decorporation treatment - Medical overview, Radiat. Prot. Dosim. 87, 51-57. 